\title{
THE THROUGH OPTIMIZATION OF FAIL-SAFE BRANCHED INJECTION TRAJECTORIES OF LAUNCH VEHICLES IN VIEW OF AERODYNAMIC LOAD CONSTRAINTS ON THE BASIS OF THE MAXIMUM PRINCIPLE
}

\author{
A.S. Filatyev and O. V. Yanova \\ Central Aerohydrodynamic Institute (TsAGI) \\ Zhukovsky 140180, Moscow Region, Russia
}

\begin{abstract}
A problem of through optimization of fail-safe branched trajectories of launchers in view of aerodynamic load constraints and restrictions on ground impact areas of separated parts (SP) is considered. The failsafety is regarded to the possibility of a recoverable vehicle (RV) to return from any point of the ascent trajectory to landing points without excess of allowable $g$-loads. So, the purpose is determination of the launcher optimal control in view of constraints on all trajectory branches: the main, corresponding to an active injection leg, and side branches, corresponding to SP fall trajectories and imaginary RV emergency trajectories, which form a continuum. The problem solution is based on the Pontryagin maximum principle (PMP).
\end{abstract}

\section{INTRODUCTION}

A problem considered is that of control optimization of a launcher injection into an orbit in view of constraints on ground impact areas of SP and fail-safety requirements (Fig. 1). Fail-safety requirements are understood as the possibility of RV staging and returning to a landing point without excess of allowable g-loads in emergency situations. Taking into account constraints on return trajectories of launcher's elements leads to consideration of branched injection trajectories, where the main branch corresponds to an active injection leg and side branches correspond to real SP falling trajectories and possible (virtual) RV emergency 


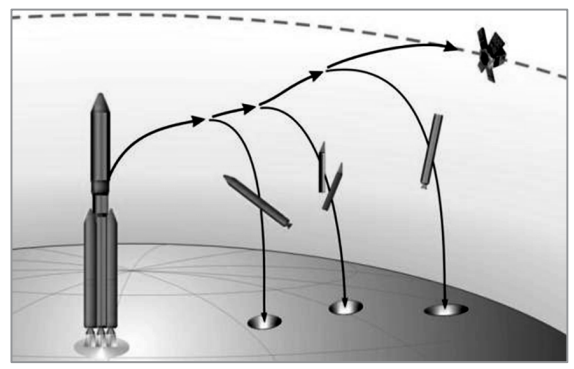

Figure 1 The scheme of a branched injection trajectory

trajectories. The RV trajectories form a continuum. Aerodynamic load constraints are taken into account at the active injection leg and RV emergency trajectories. In the last case, they define fail-safe requirements.

The solution of the optimization problem with a finite number of side branches is based on an indirect optimization method, PMP for branched processes $[1,2]$. The choice of the optimization method is determined by known advantages of the maximum principle, which are essential in solving the considered problem:

- the control program structure results from the problem solution and does not require a priori definition. This property is especially important in cases of existence of several extremals and bifurcations between them at problem parameters variations [3]. The probability of such situations rises essentially when the motion is described by differential equations with nonlinear (including discontinues) right parts; and

- a possibility of verification is put in the PMP without carrying out of additional calculation. The PMP demands solution of a conjugate system along with the state system. First integrals of state and conjugate systems as well as a physical meaning of conjugate variables as influence functions allow controlling objectively the accuracy of the calculation and the correctness of the program is realization of the method and, as consequence, the solution optimality.

Due to the PMP, the optimization problem is reduced to the two-point boundary-value problem (BVP). In this paper, some important peculiarities of the statement and solutions of the BVP are considered.

Investigations of nonequilibrium atmospheric reentry trajectories with subcircular velocities show that loads on such trajectories can essentially (in orders) increase in comparison with quasi-stationary reentry from a circular orbit $[4,5]$. One of the most vivid examples in the cosmonautics history occurred in 1975. 
Then, the third-stage engine of Soyuz-18-1 failed to start. Following the emergence separation from the launcher, the vehicle performed a suborbital flight to land safely. However, while entering dense atmospheric layers, the cosmonauts V. Lazarev and O. Makarov had to endure overloads exceeding $20 \mathrm{~g}$.

The problem of reducing loads during the nonequilibrium reentry of vehicles at subcircular velocities was also considered in publications $[6,7]$.

One of the ways to avoid a similar situation consists in formation of so-called fail-safe injection trajectories. From any point of such trajectories, an RV can reach a landing site with admissible aerodynamic loads [8]. Fail-safe trajectories can be represented as the branched ones with a continuum of side branches, each of them subjected to load constraints.

Aiming at reducing the problem to the standard optimization problem with discrete number of side branches, the method of projecting of load constraints from side branches to the branching points is used. Thus, fail-safety conditions are reduced to state constraints on the active ascent leg.

The problem solution technique has been realized in the new version of the program complex ASTER [9].

Peculiarities of the optimal solutions are considered on the example of the launcher to be developed within the framework of the Russian Federal Space Program.

\section{BASIC PROBLEM STATEMENT}

A typical branched trajectory of a launcher is shown in Fig. 1.

The motion is described by a normal system of ordinary differential equations in the initial coordinate system. On the main branch:

$$
\frac{d \mathbf{x}}{d t}=\mathbf{f}(\mathbf{x}, \mathbf{u}, t)
$$

on side branches:

$$
\frac{d \mathbf{x}_{s}}{d t}=\mathbf{f}_{s}\left(\mathbf{x}_{s}, t\right)
$$

where $\mathbf{x}=\{\mathbf{r}, \mathbf{v}, m\}^{\mathrm{T}} \in \mathbf{X}$ and $\mathbf{x}_{s}=\left\{\mathbf{r}_{s}, \mathbf{x}_{s}\right\}^{\mathrm{T}} \in \mathbf{X}_{s}$ are the state vectors on the main and side branches, respectively; $\mathbf{r}$ is the radius vector; $\mathbf{v}$ is the velocity vector; $m$ is the mass; $\mathbf{f}$ and $\mathbf{f}_{s}$ are the right part vectors on the main and side branches, respectively; the subscript " $s$ " corresponds to a side branch; $\mathbf{u} \in \mathbf{U}$ is the control vector; and $t \in\left\lfloor t_{i}, t_{f}\right\rfloor$ is the time.

The control vector is performed by the launcher axial unit vector $\mathbf{e}_{\tau}$ and thrust $T$ :

$$
\mathbf{u}=\left(\mathbf{e}_{\tau}, T\right)
$$


The boundary conditions as well as matching conditions are specified in the initial, intermediate, and final points of the branches [2]. Constraints on the control and motion of launcher elements are imposed on all branches.

The SP staging moments $\tau_{0}$ are defined by condition:

$$
Q_{0}\left(\mathbf{x}\left(\tau_{0}\right)\right)=0
$$

On the active ascent leg (the main branch), the constraints on state and control variables

$$
\mathbf{G}(\mathbf{x}, \mathbf{u}, t) \leq 0
$$

and state constraints of the first order

$$
\mathbf{O}(\mathbf{x}, t) \leq 0
$$

(including aerodynamic loads constraints) are considered.

On the right end of regular SP trajectories, the restrictions on SP ground impact points are imposed:

$$
\mathbf{Q}\left(\mathbf{x}_{s}\left(\tau_{f}\right)\right)=\mathbf{r}\left(\tau_{f}\right)-\mathbf{r}_{0}=0
$$

where the time moments $\tau_{f}$ are defined by the condition:

$$
Q_{f}\left(\mathbf{x}_{s}\left(\tau_{f}\right)\right)=h_{s}\left(\tau_{f}\right)=0 .
$$

The functional $\Phi$ is the mass injected into the specified orbit:

$$
\Phi \equiv m\left(t_{f}\right) \Rightarrow \max _{\mathbf{u}}
$$

\section{MAXIMUM PRINCIPLE CONDITIONS}

In accordance with the PMP [1], the launcher's optimal control is defined from the condition:

$$
\left\{\mathbf{e}_{\tau}, T\right\}_{\text {opt }}=\arg \max _{\left\{\mathbf{e}_{r}, T\right\}} \mathcal{H} .
$$

Here, $\mathcal{H}$ is the Hamiltonian of system (1):

$$
\mathcal{H}=\boldsymbol{\Psi}^{\mathrm{T}} \dot{\mathbf{x}}+\lambda^{\mathrm{T}} \mathbf{G}+\mu^{\mathrm{T}} \mathbf{G}_{\mathrm{ph}}
$$

where $\left(^{*}\right)=d / d t$ and $\boldsymbol{\Psi}^{\mathrm{T}}=\left\{\mathbf{P}, \mathbf{S}, P_{m}\right\}$ is the conjugate vector with components, corresponding to $\mathbf{r}, \mathbf{v}$, and $m$, respectively. Vector $\boldsymbol{\Psi}$ satisfies the equation: 


$$
\dot{\mathbf{\Psi}}=-\left(\frac{\partial \mathcal{H}}{\partial \mathbf{x}}\right)^{\mathrm{T}}
$$

with boundary conditions given in [2].

In (9), $\mathbf{G}$ is the constraint vector (4); $\mathbf{G}_{\mathrm{ph}}(\mathbf{x}, \mathbf{u}, t)=\partial \mathbf{O}(\mathbf{x}, t) / \partial t$ (see [10]) is the vector of constraints on state and control variables, derived from (5); and $\lambda$ and $\mu$ are the vectors of appropriate Lagrange multipliers.

In branching points, the conditions of the state vectors' continuity result in the following conditions of the jump of conjugate variables (transversality conditions) $[2]$ :

$$
t=\tau_{0}:\left\{\begin{array}{l}
\boldsymbol{\Psi}^{+}=\boldsymbol{\Psi}^{-}-\boldsymbol{\Psi}_{s}-\xi\left(\frac{\partial Q_{0}}{\partial \mathbf{x}^{-}}\right)^{\mathrm{T}} \\
\mathcal{H}^{+}=\mathcal{H}^{-}-\mathcal{H}_{s}
\end{array}\right.
$$

where ()$^{-}=\left.()\right|_{\tau_{0}-0} ;()^{+}=\left.()\right|_{\tau_{0}+0}$; and $Q_{0}$ is the condition of implicit definition of the branching moment $(3)$.

The constraints (6) and (7) on the SP ground impact points are displayed in the transversality conditions at the right end of side branches:

$$
t=\tau_{f}: \begin{cases}\mathbf{P}_{s}=\text { var }, & \mathbf{S}_{s}=0 \\ P_{m s}=0, & \mathcal{H}_{s}=\mathbf{P}_{s}^{\mathrm{T}} \mathbf{v}_{s}=0\end{cases}
$$

\section{BOUNDARY VALUE PROBLEM}

The application of the PMP makes it possible to reduce the optimization problem to a multipoint BVP for state (1), (2) and conjugate (10) sets of equations. In the case of the only (main) branch, the number of variable parameters of such BVP does not exceed the dimension of the state space on the main branch $n$. Each side branch increases the dimension of BVP by the dimension of the state space on the side branch $n_{s}$. Thus, in the case of $N$ side branches, the dimension of the BVP does not exceed $n_{\mathrm{BVP}}=n+n_{s} N$. However, in the particular case of boundary conditions (6) and (7), the BVP dimension can be diminished to $n_{b}=n+2 N[2]$.

The BVP solution is based on the modified Newton method [11]. The convergence is achieved by the application of the solution parameter continuation (homotopy) method [11]. The consideration of the first-order state constraints (5) results in increasing the dimension of the BVP by unity for each point of attaining a constraint (5). Peculiarities of BVP solution procedure in view of the first-order state constraints are discussed below in section 6 . 


\section{NONEQUILIBRIUM ATMOSPHERIC REENTRY PECULIARITIES}

The investigation of the nonequilibrium atmospheric reentry with subcircular velocities allows revealing a set of general features of coasting flight of aircraft in the atmosphere [4-7]:

- for the class of initial conditions under consideration, descent vehicles fail to attain the quasi-stationary gliding regimes prior to the moment of passing peak loads which, as a result, can reach excessive values. Similar regimes are realized in returning launcher SP, during test suborbital flights and in the case of emergence orbiter recovery after ascent abortion;

- on the base of direct matching analytical solutions for the state and conjugate equations, in characteristic regions with negligible and dominant influence of aerodynamic forces, the analytical estimation of the maximum overloads depending on the vehicle parameters and initial conditions is obtained; and

- the critical velocity at the reentry trajectory apogee $v_{0}$ crit, at which the reentry loads are extreme, is estimated analytically. It is proved that the critical velocity rises as the lift-to-drag ratio decreases not exceeding $1 / \sqrt{2}$ of the local circular velocity $v_{\text {circ }}$ :

$$
v_{0 \text { crit }}=\arg \max _{v_{0}} q \leq \frac{1}{\sqrt{2}} v_{\text {circ }} .
$$

And the top limit of the maximum critical velocity is reached at the returning of vehicles with zero lift-to-drag ratio.

\section{PECULIARITIES OF THE OPTIMIZATION PROBLEM WITH FAIL-SAFETY CONSTRAINTS}

In emergency situations, on the RV trajectories, aerodynamic loads constraints must be fulfilled. In particular the $g$-load constraint is imposed:

$$
G_{n}\left(\mathbf{x}_{s}(\tau)\right)=n-n_{\mathrm{adm}} \leq 0 .
$$

This is the fail-safety constraint. The fail-safe trajectory can be represented as branched ascent trajectory with a continuum of side branches in view of (11) (Fig. 2). The main peculiarity of the optimization problem is that the RV branches form continuum. 


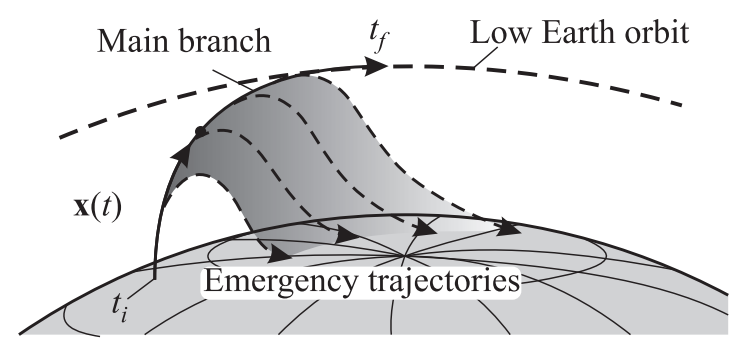

Figure 2 The scheme of a fail-safe trajectory

Analytical solutions for the state and conjugate systems [6] on the base of a loading analysis on the RV trajectories allow building the fail-safe region $\mathbf{D}$. The g-loads on the RV trajectories, initiating from the region $D$, are admissible. So, the fail-safe injection trajectory should belong to region $\mathbf{D}$ :

$$
\mathbf{x} \in \mathbf{D} \text {. }
$$

The region $D$ is built in coordinates $\left(v_{0}, h_{0}\right)$ (velocity and altitude in apogee, respectively), which can be analytically obtained (see [12]) from the state parameters in branching points $\{v, h, \gamma\}$ ( $\gamma$ is the flight-path angle).

In view of (12), fail-safety requirements (11) can be ensured by taking into account the first-order constraint on the main branch of injection trajectory:

$$
Q_{n}\left(v_{0}, h_{0}\right) \leq 0 .
$$

The constraints (12) and (13) can be represented as

$$
Q_{n}\left(v_{0}, h_{0}\right) \equiv h_{0}-h_{0 \mathrm{adm}} \leq 0
$$

where $h_{0 \text { adm }}$ corresponds to the $\mathbf{D}$-boundary $\boldsymbol{\Gamma}$ :

$$
\boldsymbol{\Gamma}=\left\{Q_{n}\left(v_{0}, h_{0}\right)=h_{0}-h_{0 \mathrm{adm}}=0\right\} .
$$

At the entry point $t_{*}$ of attaining a boundary (15) of the state constraints (13) and (14), the transversaliy conditions must be satisfied [10]:

$$
\begin{array}{r}
\boldsymbol{\Psi}^{+}-\boldsymbol{\Psi}^{-}-\nu\left(\frac{\partial Q_{n}}{\partial \mathbf{x}}\right)^{\mathrm{T}}=0 ; \\
\mathcal{H}^{+}-\mathcal{H}^{-}=\left(\Psi^{+}, \mathbf{f}^{+}\right)-\left(\Psi^{-}, \mathbf{f}^{-}\right)=0
\end{array}
$$

where $\nu$ is the Lagrange multiplier.

When the boundary (15) is attained, the optimal control is found from conditions (8) and (9) where [10]

$$
G(\mathbf{x}, \mathbf{u})=\frac{d Q_{n}}{d t}=0
$$




\section{Peculiarities of the Boundary Value Problem}

The multiplier $\nu$ (see (16)) cannot always be directly derived from conditions (16) and (17). Indeed, substituting expression (16) for the conjugate vector jump into (17), one obtains:

$$
\left(\mathbf{\Psi}^{-}, \mathbf{f}^{+}\right)-\left(\boldsymbol{\Psi}^{-}, \mathbf{f}^{-}\right)-\nu\left(\frac{\partial Q_{n}}{\partial \mathbf{x}}\right) \mathbf{f}^{+}=0
$$

However, as

$$
\left(\frac{\partial Q_{n}}{\partial \mathbf{x}}\right) \mathbf{f}^{+}=\left.\left(\frac{\partial Q_{n}}{\partial \mathbf{x}}\right) \frac{d \mathbf{x}}{d t}\right|_{t_{*}+0}=\left.\frac{d Q_{n}}{d t}\right|_{t_{*}+0}=0
$$

the transversality condition without $\nu$ follows from (17):

$$
\boldsymbol{\Psi}^{-\mathrm{T}}\left(\mathbf{f}^{+}-\mathbf{f}^{-}\right)=0 .
$$

Thus, the consideration of constraints (13) and (14) results in increasing the dimension of the BVP by unity for each point of attaining the constraint. The parameter $\nu$ is an additional variable parameter. The condition of a smooth attainment of boundary (15) can serve as a residual for its determination.

Really, the state vector $\mathbf{x}^{*}$ at point $t^{*}$ is continuous; therefore, one has:

$$
\mathbf{f}^{+}=\mathbf{f}\left(\overline{\mathbf{u}}, \mathbf{x}^{*}\right) ; \quad \mathbf{f}^{-}=\mathbf{f}\left(\mathbf{u}_{\mathrm{opt}}, \mathbf{x}^{*}\right)
$$

where $\overline{\mathbf{u}}$ :

$$
\frac{d Q_{n}}{d t}\left(\overline{\mathbf{u}}, \mathbf{x}^{*}\right)=0
$$

By considering (19), condition (18) means that

$$
\mathcal{H}\left(\overline{\mathbf{u}}, t^{*}\right)=\mathcal{H}\left(\mathbf{u}_{\mathrm{opt}}, t^{*}\right)=\max _{\mathbf{u} \in \mathbf{U}} \mathcal{H}\left(\mathbf{u}, t^{*}\right) .
$$

If the problem is correct and $\mathbf{u}_{\mathrm{opt}}$ is unambiguously governed by the condition (8), then, according to (20), one has:

$$
\mathbf{u}_{\mathrm{opt}}\left(t^{*}\right)=\overline{\mathbf{u}}\left(t^{*}\right) ; \quad \frac{d Q_{n}}{d t}\left(\mathbf{u}_{\mathrm{opt}}\left(t^{*}\right)\right)=0 .
$$

It follows from (21) that if constraints (13) and (14) are essential, function $h_{0}(t)$ is tangent to the boundary $\boldsymbol{\Gamma}$ at point $t^{*}$ in the case of optimal control (Fig. 3):

$$
\left.\frac{d h_{0}}{d t}\right|_{t^{*}-0}=0 .
$$




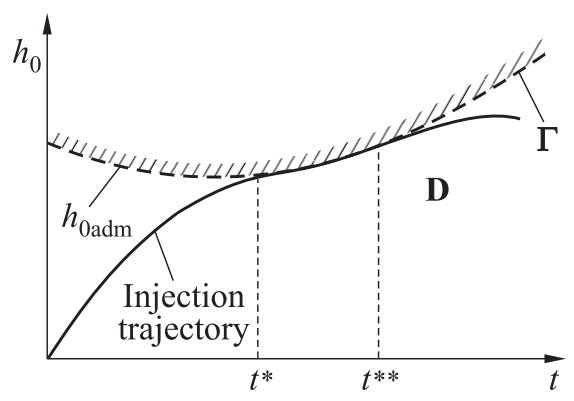

Figure 3 The scheme of a smooth entering to the boundary $\boldsymbol{\Gamma}$ of the fail-safety region $\mathbf{D}$

\section{TESTING}

The optimization technique stated above is realized in updating the program complex ASTER [9]. Before calculating, it is necessary to be convinced of the correctness of the program work. After that, the accuracy of optimum trajectory determination should be estimated. As a rule, the trajectory optimization error should not result in relative mistake of definition of the maximal inserted mass more than $10^{-5}$. It means, for example, that if the inserted mass is $20 \mathrm{t}$ (the launching mass is 600-800 t) the mistake has not to exceed a tenth part of $1 \mathrm{~kg}$. It is necessary to emphasize that the question here is not only, and not so much, the accuracy of trajectory integration (as a rule, it is 5-7 orders higher), but a total error of determination of the optimum solution. The total error depends appreciably on such factors as the accuracy of the multipoint BVP solution, the smoothness of the right parts of the motion equations, the accuracy of definition of discontinuous moments of these functions and their derivatives, and many others.

The fulfilment of these requirements is possible when using indirect methods of optimization. It is important that they are based on objective criteria, such as conservation of the first integrals, conformity of the conjugate vector to the physical sense as a function of influence of current state vector variations on the optimum functional, etc.

The changes of the Hamiltonian (see (9)) on the optimal branched injection trajectory of the vehicle are demonstrated in Fig. 4. Calculations are carried out for three integration grids: nominal, twice rarefied, and twice condensed. It is visible that fluctuations of the Hamiltonian do not exceed $10^{-7}-10^{-8}$, and the reduction of the integration step by half decreases it in one-two orders. Similar dependence on the integration step is more reliable indication of correctness of the program than a Hamiltonian value because the Hamiltonian is necessary to be compared to zero. 


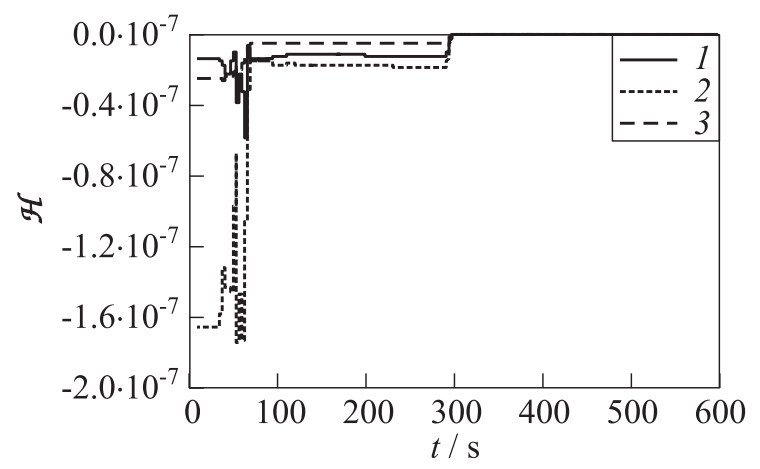

Figure 4 The Hamiltonian time-histories on the optimal injection trajectory for three integration time-grids: nominal (1); twice rarefied (3); and twice condensed (3)

The second stage of the verification of the program is to check the conformity of the conjugate variables to their physical sense as influence functions of the current state variables on the maximum inserted mass. For example, in Table 1 , the coordinates of the conjugate vector $\Psi=\{\mathbf{P}, \mathbf{S}, m\}$ are compared to corresponding coordinates of the gradient

$$
\frac{\partial m_{f \max }}{\partial \mathbf{x}}=\left\{\frac{\partial m_{f \max }}{\partial \mathbf{r}}, \frac{\partial m_{f \max }}{\partial \mathbf{v}}, \frac{\partial m_{f \max }}{\partial m}\right\}
$$

in one of characteristic points of the optimal trajectory. The gradient is calculated approximately by numerical differentiation on the basis of the central differences:

$$
\frac{\partial m_{f \max }}{\partial x_{j}} \approx \frac{m_{f \max }\left(x_{j}+\hbar\right)-m_{f \max }\left(x_{j}-\hbar\right)}{2 \hbar}
$$

where $x_{j}$ is a "disturbed" $j$-coordinate of the state vector $\mathbf{x}$. The step value $\hbar$ is selected from the condition of minimum total (methodical and rounding) errors of the numerical differentiation [13].

Table 1 The comparison of the conjugate variables to their physical sense as influence functions

\begin{tabular}{cccccccc}
\hline Function & $\mathbf{P}_{x}$ & $\mathbf{P}_{y}$ & $\mathbf{P}_{z}$ & $\mathbf{S}_{x}$ & $\mathbf{S}_{y}$ & $\mathbf{S}_{z}$ & $\mathbf{P}_{m}$ \\
\hline$\Psi$ & $1.266 \cdot 10^{-4}$ & 3.697 & $-2.290 \cdot 10^{-4}$ & 0.08496 & 0.2482 & 0 & 0.03731 \\
$-\left(\frac{\partial m_{f}}{\partial \mathbf{x}}\right)^{\mathrm{T}}$ & $1.267 \cdot 10^{-4}$ & 3.696 & $-2.319 \cdot 10^{-4}$ & 0.08497 & 0.2484 & $5.793 \cdot 10^{-8}$ & 0.03731 \\
\hline
\end{tabular}




\section{CALCULATION RESULTS}

The developed optimization technique has been applied to the calculation of the optimal branched trajectories of the two-stage launcher inserted the payload into the low Earth orbit. The calculations have been carried out taking into account the constraints:

- on the angle of attack $\alpha=0^{\circ}$ on the first-stage trajectory leg of the main branch till the moment of decreasing the dynamic pressure twice after its reaching the maximum value;

- on the pitch angle velocity $\omega \leq 4^{\circ}$;

- on the ground impact points of the first stage SP; and

- on the $g$-load at the RV emergency returning $n \leq 12$.

Initial masses of stages are as $1: 0.1042$. Initial thrust-to-weight ratios of stages are 1.378 and 0.57 , respectively. Mass ratios of separated parts to the initial launcher's mass are 0.0829 and 0.00112 , respectively. The launcher is injected to the elliptical satellite orbit with the altitude of the perigee $h_{f}$ $=135 \mathrm{~km}$ and the apogee $h_{a}=440 \mathrm{~km}$ and the inclination $i_{\text {orb }}=51.8^{\circ}$. The geographic latitude of the launch site is $51.7^{\circ}$.

The BVP dimension is $n_{b}=n+2 N+1=9$ where $n=7$ is the state vector dimension on the main branch and $N=1$ is the number of the regular side branches.

Optimal injection trajectories with and without fail-safety requirements are shown in Fig. 5: in the osculating coordinates $\left(v_{0}, h_{0}\right)$ and in the coordinates

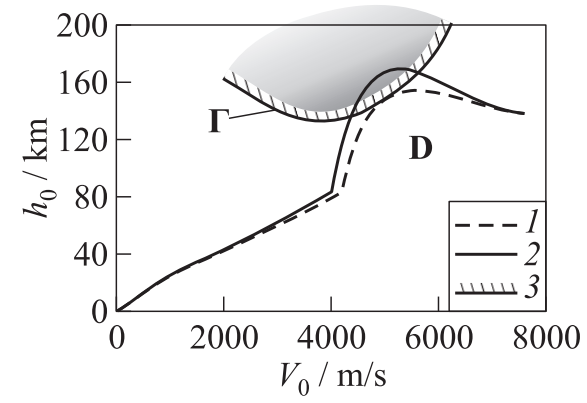

(a)

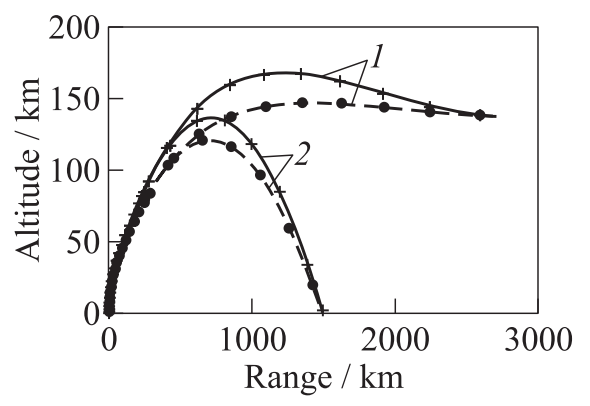

(b)

Figure 5 The optimal branched injection trajectories with (1) and without (2) fail-safety conditions in the oscillating coordinates $\left(v_{0}, h_{0}\right)$ ( 3 indicates the fail-safety constraint) $(a)$ and in the coordinates of the altitude vs. the range (solid curves - optimal trajectory; dashed curves — fail-safe optimal trajectory; 1 - ascent trajectories (main branches); and 2 - SP falling trajectories (side branches) (b) 


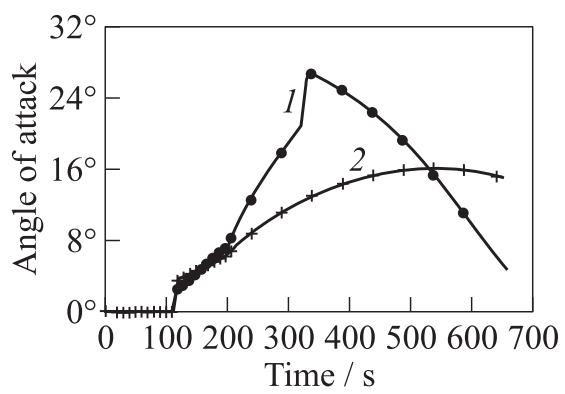

Figure 6 The optimal time-history of the angle of attack with (1) and without (2) fail-safety conditions

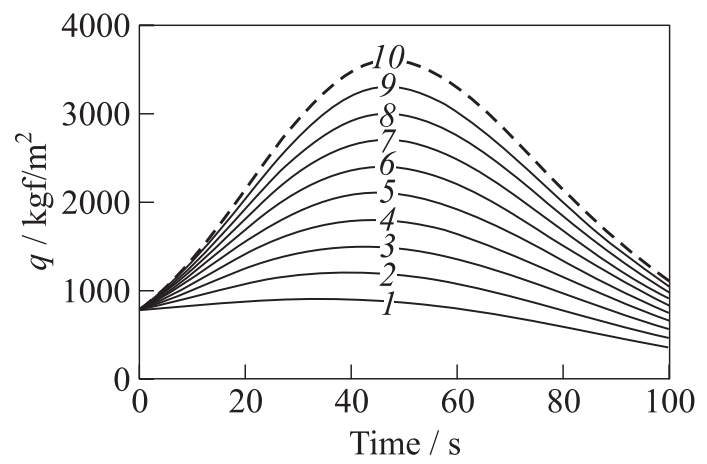

(a)

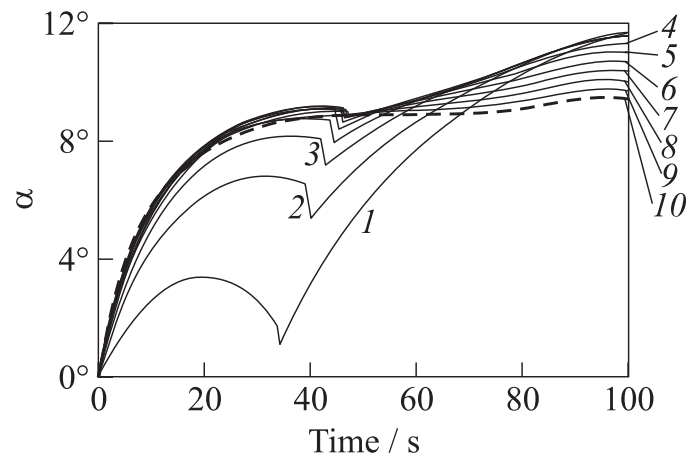

(b)

Figure 7 Modifications of the optimal time-histories of the dynamic pressure $q$ and the angle of attack $\alpha$ depending on the admissible dynamic pressure $q_{\text {adm }}$ (dependencies without constraint on the dynamic pressure are shown by dashed curves): 1 $900 \mathrm{kgf} / \mathrm{m}^{2} ; 2-1200 ; 3-1500 ; 4-1800 ; 5-2100 ; 6-2400 ; 7-2700 ; 8-$ $3000 ; 9-3300 ;$ and $10-3604 \mathrm{kgf} / \mathrm{m}^{2}$ ) 
of the altitude vs. the range. It is seen from Fig. 5 that a smooth enter to the boundary $\boldsymbol{\Gamma}$ is realized. The optimal time-history of the angle of attack on the same trajectories is shown in Fig. 6 .

The relative injected mass change at the expense of taking into account $g$-load constraint on side branches is

$$
\Delta \bar{m}_{f}=\frac{\Delta m_{f}}{m_{f \text { nom }}} \cong 0.05435
$$

where $m_{f \text { nom }}$ is the injected mass without taking into account load constraints.

Considering the state constraint on the dynamic pressure (a first-order state constraint (5)) is demonstrated with the example of the injection optimization of the SV, launching from a carrier. The initial problem parameters are as follows: $h_{i}=10 \mathrm{~km} ; v_{i}=227 \mathrm{~m} / \mathrm{s} ; \gamma_{i}$ is to be optimized; and the thrust-toweight ratio $n_{x} \approx 1.32$. Space vehicle is inserted into a circular orbit with a height of $h_{\mathrm{orb}}=115 \mathrm{~km}$ and inclination of $i_{\text {orb }}=90^{\circ}$. The results obtained are given in Fig. 7. It is seen from Fig. 7 that on the optimal trajectories with the maximum admissible dynamic pressures under study $q_{\text {adm }} \in[900,3604] \mathrm{kgf} / \mathrm{m}^{2}$, the smooth attainment of the constraint boundary is realized.

\section{CONCLUDING REMARKS}

The regular procedure of the through branched trajectory optimization on the basis of the PMP in view of the first-order state constraints is developed:

- it allows taking into account the constraints both at the main and side trajectory branches; and

- it is proved that the optimal attainment of the boundary of the first-order state constraint is realized on a tangent.

\section{ACKNOWLEDGMENTS}

The authors would like to gratefully acknowledge the financial support by the Ministry of Education and Science of the Russian Federation (Contract No. P1034).

\section{REFERENCES}

1. Pontryagin, L. S., V. G. Boltyansky, R. V. Gamkrelidze, and E. F. Mischenko. 1962. The mathematical theory of optimal processes. New York: Interscience Publs. 
2. Filatyev, A. S. 1994. Optimization of branched trajectories for aerospace transport systems. 19th ICAS Congress. Anaheim, CA. ICAS-94-5.2.3.

3. Filatyev, A.S. 2000. "Paradoxes" of optimal solutions in problems of space vehicle injection and reentry. Acta Astronautica 47(1):11-18.

4. Filatyev, A.S. 1978. Reduction of maximum dynamic and heat loads in passive motion of hypersonic vehicles using small control actions. Uchenye Zapiski TsAGI 9(2). [In Russian.]

5. Filatyev, A.S. 1980. Approximate analytical synthesis of the optimal control of hypersonic vehicles in atmospheric motion with subcircular velocities. Uchenye Zapiski TsAGI 11(1-2). [In Russian.]

6. Fllatyev, A. S. 1993. Space vehicle safety problem: Reentry with subcircular speeds. 44th Congress of the IAF. Graz, Austria. IAA 6.1-93-731.

7. Filatyev A.S. 1994. Critical reentry conditions. 19th Symposium (International) on Space Technology and Science. Yokohama, Japan. ISTS 94-c-32.

8. Filatyev, A. S. 1994. Fail-safe ascent of space transport systems. 45th Congress of the International Astronautical Federation. Jerusalem, Israel. IAF-94-IAA.6.1.676.

9. Filatyev, A.S., and O. V. Yanova. 2001. ASTER program package for the thorough trajectory optimization. AIAA Paper No. 2001-4391.

10. Bryson, A. E., Jr., and Y.C. Ho. 1975. Applied optimal control. New York: Wiley.

11. Filatyev, A.S. 1992. Optimization of spacecraft ascent using aerodynamic forces. 43rd Congress of the International Aeronautical Federation. Washington, DC. IAF92-0022.

12. Appazov, R. F., S. S. Lavrov, and V. P. Mishin. 1966. Ballistics of guided long-range missiles. Moscow: Nauka. [In Russian.]

13. Stechkin, S. B., and Yu.N. Subbotin. 1976. Splines in calculation mathematics. Moscow: Nauka. [In Russian.] 